\title{
Evaluation of Blur and Gaussian Noise Degradation in Images Using Statistical Model of Natural Scene and Perceptual Image Quality Measure
}

\author{
Anamaria BJELOPERA ${ }^{1}$, Emil DUMIC ${ }^{2}$ Sonja GRGIC ${ }^{3}$ \\ ${ }^{1}$ Dept. of Electrical Engineering and Computing, University of Dubrovnik, Cira Carica 4, 20000 Dubrovnik, Croatia \\ ${ }^{2}$ Dept. of Electrical Engineering, University North, 104. brigade 3, 42000 Varazdin, Croatia \\ ${ }^{3}$ Faculty of Electrical Engineering and Computing, Dept. of Wireless Communications, Unska 3, 10000 Zagreb, Croatia
}

anamaria.bjelopera@unidu.hr

Submitted November 1, 2017 / Accepted November 1, 2017

\begin{abstract}
In this paper we present a new method for classification of image degradation type based on Riesz transform coefficients and Blind/Referenceless Image Spatial Quality Evaluator (BRISQUE) that employs spatial coefficients. In our method we use additional statistical parameters that give statistically better results for blur and all tested degradations together in comparison with previous methods. A new method to determine level of blur and Gaussian noise degradation in images using statistical model of natural scene is presented. We defined parameters for evaluation of level of Gaussian noise and blur degradation in images. In real world applications reference image is usually not available therefore the proposed method enables classification of image degradation by type and estimation of Gaussian noise and blur levels for any degraded image.
\end{abstract}

\section{Keywords}

Image degradation, Riesz transform, BRISQUE, Gaussian noise, blur, level

\section{Introduction}

In recent years, multimedia has attracted more and more attention as a research field. Its rapid development can be seen from different aspects like content creation, delivery and finally presentation to users. With rapid development of different multimedia content, new methods are required for efficient evaluation of its quality (Quality of Experience, QoE). Quality of Experience has been defined as "the general acceptance of an application or service" (ITU P.10/G.100) [1], or as "the degree of delight or annoyance of the user of an application or service" [2].

Multimedia quality can be evaluated using different methods. Subjective quality assessment is known to be the most accurate reflection of user experience (QoE). User experience is a combination of psychophysical responses to color, motion, texture, audio and context. In a typical subjective quality assessment campaign, test subjects watch a number of original and degraded video sequences and rate their quality on a numeric scale. Subjective quality is often expressed as Mean Opinion Score (MOS) that represents a quality grade attributed by a standard average observer to a given video sequence. MOS grades are collected following well defined methods and procedures that have been proposed in the last decades, such as ITU-R BT.500-13 [3], whose goal is to ensure that the same experimental settings and conditions are used during different assessment campaigns.

However, subjective experiments are time consuming, costly and sometimes cannot be used (e.g. to constantly monitor final multimedia quality). Objective quality measures are often used during the design phase of a video communication system and in other applications where there is a constant need for assessment of video quality such as various algorithmic optimizations, content variations and transmission conditions. In general, objective quality measures can be divided into three categories according to the reference information they use:

- Full reference (FR) measures - require the original undistorted or unprocessed signal;

- Reduced-reference (RR) measures - require information derived from the original signal;

- No-reference (NR) quality - require only the processed/degraded signal.

FR objective measures require the original undistorted or unprocessed image, which makes them unsuitable for deployments in environments where real time and low complexity are required, such as real transmission systems. Because of that, RR and NR measures have been widely explored in recent years.

In this paper, we present no-reference image quality measure to determine level of blur and Gaussian noise 
degradation in images, based on statistical model of natural scene. The paper is organized as follows. Section 2 summarizes related work regarding NR image objective quality measures. Section 3 explains the new method for classification of Gaussian noise and blur degradations using statistics of Riesz transform coefficients and mean subtracted contrast normalized (MSCN) spatial coefficients. Section 4 presents a procedure for Gaussian noise and blur degradation evaluation. The conclusion is given in Sec. 5 .

\section{Related Work}

In recent years several papers propose NR image quality measures for different degradation types. Generally, in related work no-reference (NR) objective measure was developed, to grade (usually with single score) images from one or more image datasets, degraded with different degradation types. In [4], authors proposed a natural scene statistic-based distortion-generic NR image quality assessment model in the spatial domain called BRISQUE (Blind/ Referenceless Image Spatial Quality Evaluator). BRISQUE is trained and tested using five different image degradation types from LIVE image dataset [5]: JPEG2000 and JPEG compression, Gaussian noise, Gaussian blur, and a Rayleigh fast-fading channel simulation. Results show that BRISQUE is highly correlated with MOS, tested on LIVE image dataset. In [6], authors proposed a bag of featuremaps approach which avoids assumptions about the type of distortion contained in an image, focusing instead on capturing consistencies of the statistics of real world images. The measure is called Feature maps based Referenceless Image QUality Evaluation Engine (FRIQUEE). Results show that FRIQUEE is highly correlated with MOS, tested on LIVE and the Wild Image Quality Challenge Database [7], which consists of 1162 naturally distorted images that are normally captured using mobile devices under highly variable illumination conditions. In [7], authors have presented Blind Image quality assessment through anisotropy (BIQAA). This measure discriminates blur and Gaussian noise. In [9], authors presented a new model, called Oriented Gradients Image Quality Assessment (OG-IQA), showing highly competitive image quality prediction performance as compared with other quality measures. The LIVE image database [5] was used for performance evaluation of the model. In [10], authors developed an efficient general-purpose NR image quality assessment model that utilizes local spatial and spectral entropy features on distorted images, tested on LIVE image database [5] and checked on TID2008 image database [11] (among five degradation types from LIVE dataset). In [12], authors presented a novel metric for image quality assessment of blurred images. The basic idea is to estimate the point spread function (PSF) from the line spread function (LSF), whereas the LSF is constructed from edge information. The proposed metric can be used in blind image quality evaluation for real-time automatic machine vision-based applications. In [13], authors developed block-based algorithm S3 which estimates the perceived sharpness of local image regions, and which does not require the presence of edges. The measure is based on two factors: a spectral measure based on the slope of the local magnitude spectrum (S1) and a spatial measure based on local maximum total variation (S2). Unlike in previously described work in which measures give one grade per image, the so-called S3 estimator (combination of S1 and S2) can yield a local sharpness map in which greater values correspond to greater perceived sharpness within an image and across different images. S3 estimator can be used as a single measure of image sharpness. In this case the average of the $1 \%$ highest values in the S3 map is taken into account.

\section{Classification of Image Degrada- tion: Blurriness and Gaussian Noise}

The new method for classification of image degradation type combines statistics of MSCN coefficients and coefficients of Riesz transform. 18 statistical parameters calculated out of MSCN coefficients plus the same 18 statistical parameters out of coefficients of Riesz transform have been used. Besides these 36 parameters, additional parameter from Riesz transform coefficients is computed. It is standard deviation of Riesz coefficients in high frequency residual band of primary wavelet pyramid.

\subsection{Basic Properties of Riesz Transform}

Riesz transform [14], [15] connects properties of wavelet transform by means of invariance to translation and dilatation and works with steerable pyramid wavelet through rotation invariance. The selection of filter coefficients is more flexible and usually chosen for specific application.

The first property is that it is translation- and scaleinvariant as described in [14]. Riesz transform is also rotation-invariant. It is changeable with rotations. The third property of Riesz transform is inner-product preservation and it complies Parseval identity [14].

Riesz transform is also suitable for classification of types of image degradation used in our new method of classification. This new method will be based on statistical analysis of Riesz transform coefficients.

\subsection{No-reference Image Quality Measure - BRISQUE}

BRISQUE is a no-reference image quality measure which uses statistics of natural scene in the spatial domain by means of statistical analysis of mean subtracted contrast normalized (MSCN) coefficients. Among many other applications it can be used for identification of image degradation. The first step is to compute locally normalized luminances in a distorted image through local mean sub- 
traction and normalization [4]. The differences between luminance of an object and its close surroundings in relation to standard deviation are calculated. Spatial MSCN coefficients are defined as:

$$
\hat{I}(i, j)=\frac{I(i, j)-\mu(i, j)}{\sigma(i, j)+C}
$$

where $i=1,2, \ldots, M, j=1,2, \ldots, N$ are spatial indices, $C=1$ is a constant, $I(i, j)$ is image intensity, $\mu(i, j)$ is local mean and $\sigma(i, j)$ is variance.

The histograms of original images and images with different types of degradations show that some statistical properties of coefficients are changed by degradation [4]. These changes can be quantified with generalized Gaussian distribution (GGD) model and asymmetric generalized Gaussian distribution (AGGD) model. GGD is used on MSCN coefficients and AGGD on pairwise products of neighboring coefficients in horizontal $(\mathrm{H})$, vertical $(\mathrm{V})$, main-diagonal (D1) and secondary-diagonal (D2) orientations (Fig. 1).

The first two parameters calculated from GGD fit of MSCN coefficients are the parameter that describes the shape of distribution and the parameter that controls the variance. Next 16 parameters are estimated from AGGD fit of products in H, V, D1 and D2 orientations, i.e. four parameters are calculated per orientation. These parameters are: parameter that controls the shape of distribution, left and right variance (scale parameters) that control the spread on each side of the mode and mean value [4]. The total of 36 parameters is consisted of 18 parameters calculated on the original image scale and 18 on a reduced resolution. For mapping from feature space to quality scores LIBSVM packet [16] is used.

\subsection{Method for Classification of Image Degra- dation Type}

For the Riesz transform number of scales and filters should be defined. Riesz wavelet tool from [17] generates coefficients of Q structure and coefficients of high frequency residual band R. Q structure is composed of cells of matrices. Every element of the cell corresponds to one wavelet scale. Three different filters are chosen: the first wavelet filter is a vertical line detector, the second a diagonal line detector, and the third a horizontal line detector. For the calculation of Riesz coefficients, scale of two was chosen. Q structure is composed of three cells: $Q\{1,1\}$ cell composed of elements that correspond to scale two, Q $\{1,2\}$ cell whose elements correspond to scale one and $\mathrm{Q}\{1,3\}$ cell which defines elements of the original image. For each scale, elements of image are filtered with each of three early mentioned filters (Fig. 2). If dimensions of an image are for example $500 \times 500, \mathrm{Q}\{1,1\}$ cell is consisted of $500 \times 500 \times 3$ elements (each filter is used for $500 \times 500$ elements of image), $\mathrm{Q}\{1,2\}$ cell contains $250 \times 250 \times 3$ elements and $\mathrm{Q}\{1,3\}$ cell has $125 \times 125 \times 3$ elements.

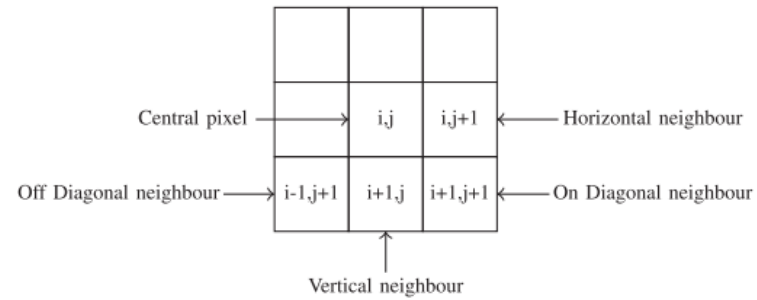

Fig. 1. Paired products along four orientations (H, V, D1 and D2) at distance of one pixel used for calculating neighboring statistical relationships [4].

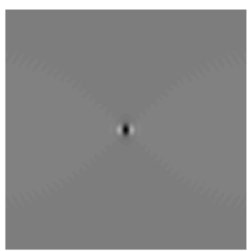

a) Filter1

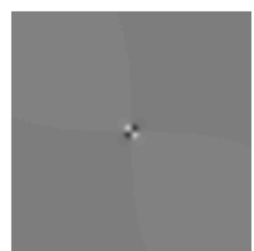

b) Filter 2

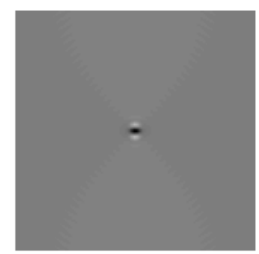

c) Filter 3
Fig. 2. Filters used for Riesz transform.

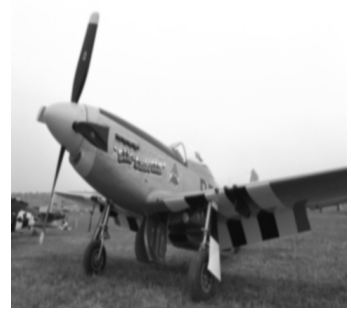

a) Image Plane with blur

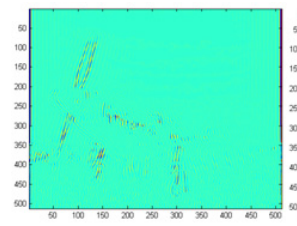

b) $\mathrm{Q}\{1,1\}$-filter 1

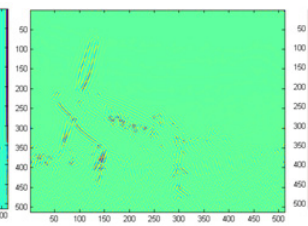

c) $\mathrm{Q}\{1,1\}$-filter 2

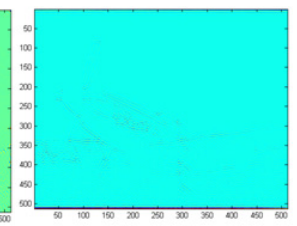

d) $\mathrm{Q}\{1,1\}$-filter 3
Fig. 3. Q $\{1,1\}$ cell for image Plane with blur degradation.

$\mathrm{Q}\{1,1\}$ elements filtered with the third filter are chosen for calculation of 18 statistical parameters out of Riesz transform coefficients early mentioned.

In Fig. 2 channels (filters) for Riesz transform are shown. The channels of $\mathrm{Q}\{1,1\}$ cell for image Plane with blur degradation are shown in Fig. 3.

37 parameters are used in LIBSVM in order to determine the image degradation type. BRISQUE Matlab tool from [18] was used for calculation of MSCN coefficients and tool from [17] to calculate Riesz wavelet coefficients.

To test the effectiveness of the proposed method the image database consisted of distorted images was divided into two different groups: the training (about $80 \%$ of all images) and the testing group (about $20 \%$ of all images). 37 parameters were extracted from early mentioned distorted images and then used for LIBSVM regressor using train-test procedure. This was repeated for 1000 iterations 
and then mean value was calculated. This was also repeated for BRISQUE method and then these two methods were compared using McNemar's statistical test [19] for LIVE database [20] for different types of degradation: JPEG compression, JPEG2000 compression, Gaussian blur and white Gaussian noise. McNemar's test calculates statistical significance of two methods i.e. whether one of the method is statistically more significant that the other one. We used significance level of 5\%.

Table 1 shows the results of statistical significance between BRISQUE method and the new method for classification of image degradation type. ' + ' means that measure gives statistically better results, '-' means that measure gives statistically worse results, and ' $x$ ' means that results are statistically similar in both measures.

Table 1 shows that our method shows statistically better results than BRISQUE method for Gaussian noise and all four types of image degradations when used together i.e. JPEG2000, JPEG, Gaussian blur and Gaussian noise. The accuracy of determining class of Gaussian noise distortion with the new method was $98.32 \%$ and the accuracy for Gaussian blur was $93.60 \%$. When all four distortions were used together the accuracy was $90.83 \%$.

The numbers in Tab. 2 are numbers of images. For example, the number 30464 in the first row and column is the number of images that have JPEG2000 degradation and were classified as JPEG2000, the number 3422 in the second row and the first column is the number of images that have JPEG2000 degradation and were categorized as images with JPEG degradation. Then number 156 in the

\begin{tabular}{|l|c|c|c|c|}
\hline \multirow{2}{*}{ LIVE } & \multicolumn{2}{|c|}{ McNemar's exact test } & \multicolumn{2}{c|}{$\begin{array}{c}\text { McNemar's } \\
\text { Hi-squared test }\end{array}$} \\
\cline { 2 - 5 } & $\begin{array}{l}\text { BRISQUE } \\
\text { method }\end{array}$ & $\begin{array}{l}\text { New } \\
\text { method }\end{array}$ & $\begin{array}{l}\text { BRISQUE } \\
\text { method }\end{array}$ & $\begin{array}{l}\text { New } \\
\text { method }\end{array}$ \\
\hline $\begin{array}{l}\text { Gaussian } \\
\text { noise }\end{array}$ & - & + & - & + \\
\hline $\begin{array}{l}\text { Gaussian } \\
\text { blur }\end{array}$ & + & - & + & - \\
\hline $\begin{array}{l}\text { All } \\
\text { distortions } \\
\text { (4 types) }\end{array}$ & - & + & - & + \\
\hline
\end{tabular}

Tab. 1. McNemar's test for 1000 iterations for LIVE database.

\begin{tabular}{|l|c|c|c|c|}
\hline \multirow{2}{*}{$\begin{array}{c}\text { Resolved } \\
\text { degradation }\end{array}$} & \multicolumn{4}{|c|}{ Real degradation } \\
\cline { 2 - 5 } & JPEG2000 & JPEG & $\begin{array}{l}\text { Gaussian } \\
\text { noise }\end{array}$ & $\begin{array}{l}\text { Gaussian } \\
\text { blur }\end{array}$ \\
\hline JPEG2000 & 30464 & 3526 & 10 & 1281 \\
\hline JPEG & 3422 & 31083 & 410 & 420 \\
\hline $\begin{array}{l}\text { Gaussian } \\
\text { noise }\end{array}$ & 156 & 579 & 29495 & 218 \\
\hline $\begin{array}{l}\text { Gaussian } \\
\text { blur }\end{array}$ & 929 & 991 & 85 & 28081 \\
\hline $\begin{array}{l}\text { Total } \\
\text { number of } \\
\text { images }\end{array}$ & 34971 & 36179 & 30000 & 30000 \\
\hline
\end{tabular}

Tab. 2. The accuracy of classification of image degradation for 1000 iterations for LIVE database. third row and the first column is the number of images that have JPEG2000 degradation and were classified as images with Gaussian noise, and the last number 929 in the fourth row and the first column is the number of images that have JPEG2000 degradation and were resolved as images with Gaussian blur. The results in Tab. 2 show that using the new proposed method for classification, Gaussian noise was most common wrongly classified as JPEG and Gaussian blur as JPEG2000 degradation.

Statistical McNemar's test was performed using testing group consisted of images from LIVE database and training group consisted of images from VCL@FER database [21].VCL@FER database also contains images with commonly used JPEG2000 compression, JPEG compression, Gaussian noise and blur. ' + ' , '-' and ' $x$ ' in Tab. 3 have the same meanings as in Tab. 1.

Table 3 shows the case when the training is performed on LIVE database and the testing on VCL@FER database. The new method achieves statistically significantly better results for images degraded with blur.

The comparison of the new method and BRISQUE for images degraded with Gaussian noise shows that results of both methods are very similar. None of the methods exhibited statistically better results. For statistical testing when all four types of degradation are used together, the new method showed statistically significantly better results.

The accuracy of class determination for Gaussian noise distortion was $87.68 \%$ with the new method while the accuracy of class determination for Gaussian blur was

\begin{tabular}{|l|c|c|c|c|}
\hline \multirow{2}{*}{} & \multicolumn{2}{|c|}{ McNemar's exact test } & \multicolumn{2}{c|}{$\begin{array}{c}\text { McNemar's } \\
\text { Hi-squared test }\end{array}$} \\
\cline { 2 - 5 } & $\begin{array}{l}\text { BRISQUE } \\
\text { method }\end{array}$ & $\begin{array}{l}\text { New } \\
\text { method }\end{array}$ & $\begin{array}{l}\text { BRISQUE } \\
\text { method }\end{array}$ & $\begin{array}{l}\text { New } \\
\text { method }\end{array}$ \\
\hline $\begin{array}{l}\text { Gaussian } \\
\text { noise }\end{array}$ & $\times$ & $\times$ & $\times$ & $\times$ \\
\hline $\begin{array}{l}\text { Gaussian } \\
\text { blur }\end{array}$ & - & + & - & + \\
\hline $\begin{array}{l}\text { All } \\
\text { distortions } \\
\text { (4 types) }\end{array}$ & - & + & - & + \\
\hline
\end{tabular}

Tab. 3. McNemar's test for the training on LIVE database and the testing on VCL@FER database.

\begin{tabular}{|l|c|c|c|c|}
\hline \multirow{2}{*}{$\begin{array}{c}\text { Resolved } \\
\text { degradation }\end{array}$} & \multicolumn{4}{|c|}{ Real degradation } \\
\cline { 2 - 5 } & JPEG2000 & JPEG & $\begin{array}{l}\text { Gaussian } \\
\text { noise }\end{array}$ & $\begin{array}{l}\text { Gaussian } \\
\text { blur }\end{array}$ \\
\hline JPEG2000 & 113 & 3 & 2 & 52 \\
\hline JPEG & 15 & 127 & 13 & 10 \\
\hline $\begin{array}{l}\text { Gaussian } \\
\text { noise }\end{array}$ & 2 & 2 & 121 & 2 \\
\hline $\begin{array}{l}\text { Gaussian } \\
\text { blur }\end{array}$ & 8 & 6 & 2 & 74 \\
\hline $\begin{array}{l}\text { Total image } \\
\text { number }\end{array}$ & 138 & 138 & 138 & 138 \\
\hline
\end{tabular}

Tab. 4. The accuracy of classification of image degradation for the training on LIVE database and the testing on VCL@FER database. 
$53.62 \%$. For all four distortions used together it was $78.80 \%$.

Table 4 presents the results of the new proposed method. Gaussian noise was the most common wrongly classified as JPEG and Gaussian blur was the most common wrongly classified as JPEG2000 degradation. The results can be interpreted in the same way as the results in Tab. 2.

\section{Level of Blurriness and Gaussian Noise}

The proposed method for evaluation of level of blurriness and Gaussian noise is based on certain parameters of statistical analysis of MSCN coefficients described in Sec. 3.2. The level of Gaussian noise will be defined by standard deviation of noise and the level of blurriness by standard deviation of blur.

For evaluation of Gaussian noise level in image, VCL@FER database was used. Six standard deviations of Gaussian noise were calculated for six levels of degradation of images with Gaussian noise degradation. The values of 18 parameters of BRISQUE measure calculated for images with Gaussian noise degradation on VCL@FER database were analyzed to identify regularities for six levels of degradation. It was noticed that few parameters are sensitive to level of Gaussian noise and have regularities i.e. their values were increasing when the level of Gaussian noise was increased. The calculated intervals of parameter values for each level of Gaussian noise degradation were defined with "mean value \pm standard deviation of the parameter value". The intervals should be non-overlapped. The regularity was noticed for the following parameters: the variance of MSCN coefficients (parameter 2 in [4]), the left variance for D1 diagonal products of MSCN coefficients (parameter 13 [4]) and the right variance for D2 diagonal products of MSCN coefficients (parameter 18 [4]). The optimal curves that should fit mean values of each parameter $(2,13$ and 18) for each level of Gaussian noise degradation were found by Curve Fitting Matlab Tool, (2)-(4). The curve fits are used for determination of parameter values.

The curve fit for parameter 2 is:

$$
\begin{array}{ll}
f(x)=\left(p_{1} x^{2}+p_{2} x+p_{3}\right) /\left(x+q_{1}\right), \\
p_{1}=8.219 \cdot 10^{-5} & (-0.000513,0.0006774), \\
p_{2}=0.8134 & (0.7834,0.8434), \\
p_{3}=2.121 & (1.828,2.414), \\
q_{1}=7.73 & (6.814,8.646) .
\end{array}
$$

The curve fit for parameter 13 is:

$$
\begin{aligned}
& f(x)=a \mathrm{e}^{b x}+c \mathrm{e}^{d x} \\
& a=0.3878 \quad(0.2726,0.503) \\
& b=0.01077 \quad(0.002142,0.0194), \\
& c=-0.2737 \quad(-0.3761,-0.1714), \\
& d=-0.15 \quad(-0.2764,-0.02368)
\end{aligned}
$$

The curve fit for parameter 18 is:

$$
\begin{array}{ll}
f(x)=a \mathrm{e}^{b x}+c \mathrm{e}^{d x}, \\
a=0.4095 \quad(0.2208,0.5982), \\
b=0.002657 \quad(-0.007877,0.01319), \\
c=-0.3429 \quad(-0.5197,-0.1661), \\
d=-0.08564 \quad(-0.144,-0.02731) .
\end{array}
$$

Table 5 shows the standard deviation values of each level of Gaussian noise degradation.

To evaluate level of Gaussian noise degradation for any image, parameters 2, 13 and 18 are calculated. Then for each calculated parameter value, the level of Gaussian noise degradation is determined.

The level of Gaussian noise degradation was determined as an average of three calculated levels of each parameter. The equation for evaluation of levels is:

$$
\text { Evaluated level }=\frac{L 2+L 13+L 18}{3}
$$

where $L 2$ is the evaluated level for parameter $2, L 13$ for parameter 13 , and $L 18$ for parameter 18 .

Figure 4 shows the image Womanhat as original and with the $4^{\text {th }}$ level of Gaussian noise which is also recognized as level 4 with our new method.

For evaluation of blur, images from LIVE database were used to get suitable description of 18 parameters of BRISQUE measure. Six standard deviations of blur were calculated for six levels of blur degradations. The BRISQUE parameter values were analyzed in order to identify regularities for six different levels of blur degradation. It was noticed that few parameters are sensitive to the level of blur degradation. When the level of blur degra-

\begin{tabular}{|l|c|c|c|c|c|c|}
\hline $\begin{array}{l}\text { Level of } \\
\text { degradation }\end{array}$ & 1 & 2 & 3 & 4 & 5 & 6 \\
\hline $\begin{array}{l}\text { Standard } \\
\text { deviation }\end{array}$ & 1.0748 & 4.0374 & 7.9298 & 15.9059 & 27.4920 & 37.2592 \\
\hline
\end{tabular}

Tab. 5. The calculated standard deviations for images from VCL@FER database for six levels of Gaussian noise degradation.

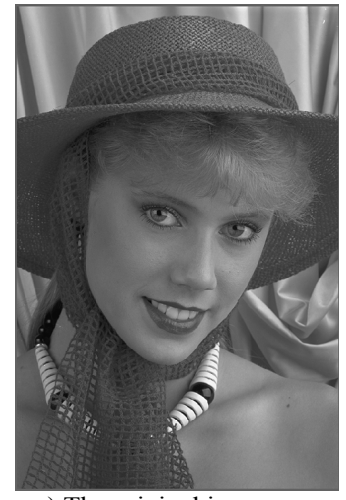

a) The original image

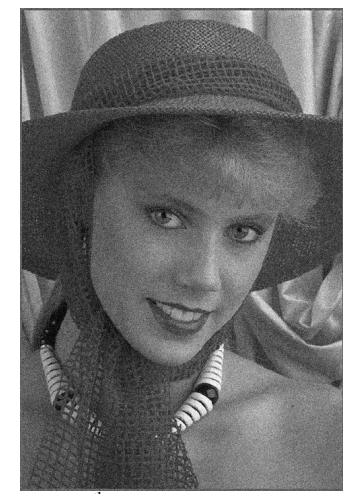

b) The $4^{\text {th }}$ level of Gaussian noise
Fig. 4. Image Womanhat from LIVE database with the $4^{\text {th }}$ level of Gaussian noise degradation that was evaluated as level 4 with our new method. 
dation was increasing, the parameter values were decreasing. Similar as for Gaussian noise degradation the calculated intervals of parameter values for each level of blur degradation were defined with "mean value \pm standard deviation of the parameter value". The regularity was noticed for the following parameters: the variance of MSCN coefficients using GGD (parameter 2 [4]), the right variance for vertical products of MSCN coefficients (parameter 10 [4]) and the right variance for D2 diagonal products of MSCN coefficients (parameter 18 [4]) using AGGD. The optimal curves to fit mean values of each parameter $(2,10$ and 18) for each level of blur degradation (defined in (6), (7) and (8)) were found using the same Matlab Tool as for Gaussian noise. The curve fits are used for determination of parameter values. Parameters 2 and 18 used for blur are used for Gaussian noise, too, but their behavior for blur is different so the curves are also going to be different.

The curve fit for parameter 2 is:

$$
\begin{array}{ll}
f(x)=a \mathrm{e}^{b x}+c \mathrm{e}^{d x}, \\
a=0.5662 & (0.5319,0.6005), \\
b=-2.15 & (-2.549,-1.752), \\
c=0.08353 & (0.05323,0.1138), \\
d=0.05449 & (-0.01875,0.1277) .
\end{array}
$$

The curve fit for parameter 10 is:

$$
\begin{aligned}
& f(x)=a \mathrm{e}^{b x}+c \mathrm{e}^{d x}, \\
& a=0.3028 \quad(0.2848,0.3206), \\
& b=-2.141 \quad(-2.495,-1.766), \\
& c=0.0144 \quad(0.001278,0.02752), \\
& d=0.1395 \quad(-0.04372,-0.3227) .
\end{aligned}
$$

The curve fit for parameter 18 is:

$$
\begin{array}{ll}
f(x)=a \mathrm{e}^{b x}+c \mathrm{e}^{d x}, \\
a=0.2168 & (0.1651,0.2684), \\
b=-2.806 & (-3.835,-1.777), \\
c=0.02966 & (0.01237,0.04695), \\
d=-0.06946 & (-0.1934,0.05447) .
\end{array}
$$

Table 6 shows the standard deviation values for each level of blurriness.

The parameters 2, 10 and 18 can be calculated for any image to evaluate level of blurriness. Then for each calculated value of parameter, the level of blurriness is determined. The level of blurriness was determined as an average of three calculated levels of each parameter. The equation for evaluation of levels is:

$$
\text { Evaluated level }=\frac{L 2+L 10+L 18}{3}
$$

where $L 2$ is the evaluated level for parameter 2, $L 10$ for parameter 10 , and $L 18$ for parameter 18 .

\begin{tabular}{|l|c|c|c|c|c|c|}
\hline $\begin{array}{l}\text { Level of } \\
\text { degradation }\end{array}$ & 1 & 2 & 3 & 4 & 5 & 6 \\
\hline $\begin{array}{l}\text { Standard } \\
\text { deviation }\end{array}$ & 0.4 & 0.5 & 0.6 & 0.8 & 1.0 & 5.0 \\
\hline
\end{tabular}

Tab. 6. The calculated standard deviations for images from LIVE database for six levels of blur degradation.

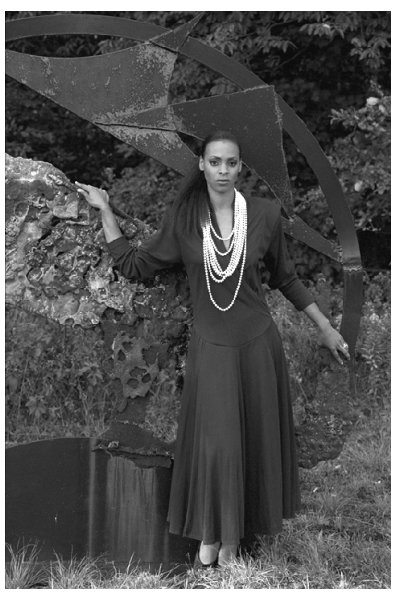

a) The original image

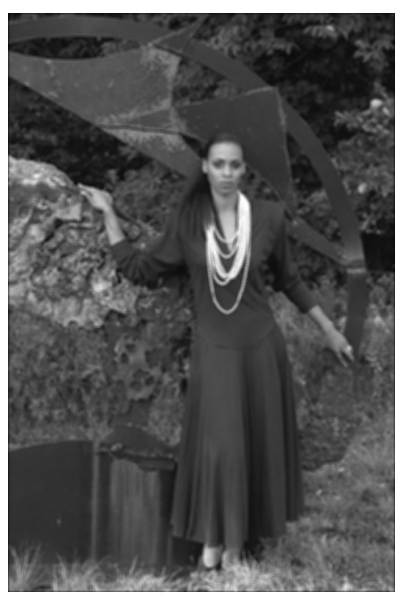

b) The $6^{\text {th }}$ level of blurrines
Fig. 5. Image Woman from LIVE database with the $6^{\text {th }}$ level of blur degradation that was evaluated as level 6 with our new method.

Figure 5 shows the image Woman as original and with the $6^{\text {th }}$ level of blur which is also recognized as level 6 with our new method.

Additionally, we tested the performance of the proposed quality measure using Pearson's and Spearman's correlation between DMOS (Difference MOS) LIVE dataset scores for noise degradation, MOS VCL@FER dataset scores for blur degradation and results of the proposed measure. LIVE dataset consists of 145 noisy images, while VCL@FER dataset consists of 138 blurred images. The Pearson's correlation was calculated after the application of a nonlinear regression step using a 5-parameter logistic function as recommended in [22]:

$$
Q(z)=b_{1} \cdot\left\{\frac{1}{2}-\frac{1}{1+\exp \left[b_{2} \cdot\left(z-b_{3}\right)\right]}\right\}+b_{4} \cdot z+b_{5} .
$$

For Gaussian noise degradation (using LIVE dataset) we obtained Pearson's correlation of 0.9686 and Spearman's correlation of 0.9752 . For blur degradation (using VCL@FER dataset) we obtained Pearson's correlation of 0.7657 and Spearman's correlation of 0.7237 . As it can be seen from Tab. 2 and 4, blur versus noise degradation can be accurately differentiated, so we assumed perfect classification.

For comparison, peak signal-to-noise ratio (PSNR) correlation with DMOS in LIVE dataset for Gaussian noise is 0.9880 (Pearson's correlation) and 0.9854 (Spearman's correlation). PSNR correlation with MOS in VCL@FER dataset for blur degradation is 0.7868 (Pearson's correla- 
tion) and 0.7791 (Spearman's correlation). PSNR is a full reference measure where the original image is available while the new measure is a no-reference measure so results for the new measure are good enough.

\section{Conclusion}

The purpose of this paper is to find a suitable way of classification of image degradation type and to evaluate the level of Gaussian noise and blur image degradations. Usually in real-life applications there is no reference image for a degraded image so it is not an easy task to find which type and level of degradation exists in the image. The idea is to find the type and level of degradation for any degraded image without knowing the original one.

The new method for classification of image degradation which is based on BRISQUE no-reference quality measure and Riesz transform is proposed. Riesz transform has properties of rotation, translation and scale invariation, and coefficients of the used filters can be chosen more flexibly in comparison with wavelet transform. BRISQUE quality measure shows statistically better results than most standard full-reference and no-reference quality measures. The method uses statistical analysis of MSCN coefficients and quantifies the loss of natural properties of images when certain degradation in the image exists. 18 parameters are calculated out of MSCN coefficients and 19 out of Riesz transform coefficients. These 37 parameters in total are used in support vector machine LIBSVM regressor in order to determine the type of image degradation. This new method can resolve four types of degradation: JPEG2000 compression, JPEG compression, blur and Gaussian noise. We have shown McNemar's statistical test results for Gaussian noise, blur and a case when all four types of degradation are used together. This test compares BRISQUE and our new method when the training and the testing is made on images in the same LIVE database and when the training is done on images from LIVE database and the testing on images from VCL@FER database. It is concluded that when testing and training is done on images in different databases the new method shows better results for blur. For Gaussian noise none of the methods was presented as statistically more significant and both methods had similar results. When using all four of types of degradation together, the new method showed better results.

The new method for evaluation of the level of Gaussian noise and blur is also proposed. For each degradation (Gaussian noise and blur), three parameters were selected to evaluate the level of blurriness.

The performance of the proposed quality measure was tested using Pearson's and Spearman's correlation with DMOS in LIVE dataset and MOS in VCL@FER dataset. Results of the proposed NR measure were comparable to full reference quality measure.

When the class of image degradation is determined and the level of degradation is evaluated, it is possible to use this knowledge for image enhancement and removal of degradations in any image without knowing the original image.

\section{References}

[1] ITU-R P.10/G.100 Vocabulary for performance and quality of service. International Telecommunication Union/ITU Radiocommunication Sector, 2006.

[2] LE CAllet, P., MOELleR, S., PERKINS, A. (eds.) Qualinet White Paper on Definitions of Quality of Experience. In COST Action IC 1003, Laussane (Switzerland), 2012.

[3] ITU-R BT.500-13 Methodology for the subjective assessment of the quality of television pictures. International Telecommunication Union/ITU Radiocommunication Sector, 2012.

[4] MITTAL, A., MOORTHY, A. K., BOVIK, A. C. No-reference image quality assessment in the spatial domain. IEEE Transactions on Image Processing, 2012, vol. 21, no. 12, p. 4695-4708. DOI: 10.1109/TIP.2012.2214050

[5] SHEIKH, H. R., SABIR, M. F., BOVIK, A. C. A statistical evaluation of recent full reference image quality assessment algorithms. IEEE Transactions on Image Processing, 2006, vol. 15 , no. 11, p. 3440-3451. DOI: 10.1109/TIP.2006.881959

[6] GHADIYARAM, D., BOVIK, A. C. Perceptual quality prediction on authentically distorted images using a bag of features approach. Journal of Vision, 2017, vol. 17, no. 1:32, p. 1-25. DOI: $10.1167 / 17.1 .32$

[7] GHADIYARAM, D., BOVIK, A. C. Massive online crowdsourced study of subjective and objective picture quality. IEEE Transactions on Image Processing, 2016, vol. 25, no. 1, p. 372-387. DOI: 10.1109/TIP.2015.2500021

[8] GABARDA, S., CRISTOBAL, G. Blind image quality assessment through anisotropy. Journal of the Optical Society of America, 2007, vol. 24, no. 12, p. B42-B51. DOI: 10.1364/JOSAA.24.000B42

[9] LIU, L., HUA,Y., ZHAO, Q., HUANG, H., BOVIK, A. C. Blind image quality assessment by relative gradient statistics and adaboosting neural network. Signal Processing: Image Communication, 2016, vol. 40, no. 1, p. 1-15. DOI: 10.1016/j.image.2015.10.005

[10] LIU, L., LIU, B., HUANG, H., BOVIK, A. C. No-reference image quality assessment based on spatial and spectral entropies. Signal Processing: Image Communication, 2014, vol. 29, no. 8, p. 856 to 863. DOI: $0.1016 /$ j.image.2014.06.006

[11] PONOMARENKO, N., LUKIN, V., ZELENSKY, A., et al. TID2008 - A database for evaluation of full-reference visual quality assessment metrics. Advances of Modern Radioelectronics, 2009, vol. 10, p. 30-45. Available at: http: //www.ponomarenko.info/tid2008.htm/

[12] WU, S., XIE, S., LIN, W. Blind measurement of image blur for vision-based applications. Chapter in: Lin, W., Tao, D., Kacprzyk, J., et al. (eds.) Multimedia Analysis, Processing and Communications. Studies in Computational Intelligence. Heidelberg, Berlin: Springer, 2011, vol. 346, p. 185-215. DOI: 10.1007/978-3-64219551-8_6

[13] VU, C. T., PHAN, T. D., CHANDLER, D. M. S : A spectral and spatial measure of local perceived sharpness in natural images. IEEE Transaction on Image Processing, 2012, vol. 21, no. 3, p. 934-945. DOI: 10.1109/TIP.2011.2169974

[14] UNSER, M., VAN DE VILLE, D. Wavelet steerability and the higher-order Riesz transform, IEEE Transactions on Image Processing, 2010, vol. 19, no. 3, p. 636-652. DOI: 10.1109/TIP.2009.2038832 
[15] BJELOPERA, A., DUMIC, E., GRGIC, S. Classification of image degradation using Riesz transform. In Proceedings of the $23 \mathrm{rd}$ International Conference on Systems, Signals and Image Processing IWSSIP 2016. Bratislava (Slovakia), 2016. DOI: 10.1109/IWSSIP.2016.7502741

[16] LIBSVM Software [Online] Cited 2017-08-20. Available at: https://www.csie.ntu.edu.tw/ cjlin/libsvm/

[17] Riesz transform: [Online] Cited 2017-08-20. Available at http://bigwww.epfl.ch/demo/steerable-wavelets/

[18] BRISQUE Software: [Online] Cited 2017-08-20. Available at: http://live.ece.utexas.edu/research/quality/

[19] McNemar test: [Online] Cited 2017-09-01. Available at: http://www.mathworks.com/matlabcentral/fileexchange/6297mcnemarextest/content/McNemarextest.m

[20] LIVE IQA database: [Online] Cited 2017-08-20. Available at: http://live.ece.utexas.edu/research/quality/subjective.htm

[21] VCL@FER database: [Online] Cited 2017-08-20. Available at: http://www.vcl.fer.hr/quality/vclfer.html

[22] SHEIKH, H. R. Image quality assessment using natural scene statistics. Ph.D. Dissertation. University of Texas at Austin, 2004. $231 \mathrm{p}$.

\section{About the Authors ...}

Anamaria BJELOPERA is a postdoctoral researcher at the Dept. of Electrical Engineering and Computing, Uni- versity of Dubrovnik. She received her Ph.D. degree in Electrical Engineering from the University of Zagreb in 2016. Her current research interests include subjective and objective image quality evaluation methods, image degradation classification and removal, Riesz transform, different DVB standards and television signal transmission.

Emil DUMIC is an Assistant Professor at the University North, Dept. of Electrical Engineering. He received his $\mathrm{Ph} . \mathrm{D}$. degree from the University of Zagreb, Faculty of Electrical Engineering and Computing in 2011. His current research interests include development of objective image and video quality measures, subjective and objective assessments on image, video and $3 \mathrm{D}$ video databases, image interpolation, impact of different channel models on bit error rate in different DVB standards, etc.

Sonja GRGIC received her Ph.D. degree in Electrical Engineering from the University of Zagreb in 1996, where she is currently a Professor. Her research interests include image quality evaluation methods, television signal transmission, video communication technologies and image forensics. She is the author of 5 book chapters, 20 scientific journal papers, more than 140 papers published on international scientific conferences as well as of 15 reviewed studies and expert works. She was the editor of 8 international conference proceedings. 